\title{
MRAC Revisited: Guaranteed Performance with Reference Model Modification
}

\author{
Vahram Stepanyan and Kalmanje Krishnakumar
}

\begin{abstract}
This paper presents modification of the conventional model reference adaptive control (MRAC) architecture in order to achieve guaranteed transient performance both in the output and input signals of an uncertain system. The proposed modification is based on the tracking error feedback to the reference model. It is shown that approach guarantees tracking of a given command and the ideal control signal (one that would be designed if the system were known) not only asymptotically but also in transient by a proper selection of the error feedback gain. The method prevents generation of high frequency oscillations that are unavoidable in conventional MRAC systems for large adaptation rates. The provided design guideline makes it possible to track a reference command of any magnitude form any initial position without re-tuning. The benefits of the method are demonstrated in simulations.
\end{abstract}

\section{INTRODUCTION}

The asymptotic behavior of adaptive systems is well researched during last couple of decades, and it is well known that the asymptotic tracking can be always achieved using so called Lyapunov redesign method. However, the transient behavior of the input and output signals can be very oscillatory with big excursions [13]. There has been great deal of efforts to modify the control architecture and the adaptive laws from the perspective of improving the transient behavior of the tracking error. The majority of these efforts led to high gain linear nonadaptive feedback [3], [4], [12], switching control law [8], [9] or to a parameter dependent persistent excitation condition [1].

In general, increasing the adaptation rate while reducing the tracking error magnitude (see for example [6]), generates high frequency oscillations and big overshoot in the control signal leading to possible actuator failures or excitation of unmodeled dynamics, which in turn can drive the overall system to instability. This shortcoming is common for the majority of existing adaptive control methods. Recently some results appear in the control community explicitly addressing the input signal transient behavior, which is very important from the point of view of the performance specifications of closed loop systems.

Vahram Stepanyan is with Mission Critical Technologies Inc. at NASA Ames Research Center, Moffett Field, CA 94035, email: vahram.stepanyan@nasa.gov

Kalmanje Krishnakumar is with Intelligent Systems Division, NASA Ames Research Center, Moffett Field, CA 94035, email: kalmanje.krishnakumar@nasa.gov
In [2] the authors proposed a new adaptive control architecture, called $L_{1}$ adaptive control, that can achieve close tracking of a given reference command both in transient and steady state by increasing the adaptation rate. The low pass filter introduced in the control channel prevents the high frequency oscillations in the control signal that closely follows the ideal one. However, the approach loses the possibility to have a real reference model to track, for which the control metrics can be specified.

An alternative approach is proposed in [11] for a class of multi-input multi-output uncertain nonlinear systems to track a given reference model. Along with the tracking error it uses also its integral to guarantee the transient performance of both input and output signals. The control algorithm internally generates a low pass filter, thus preventing high frequency oscillations for the large adaptation rates.

In this paper we take somewhat different approach and instead of modifying the control architecture or the adaptive laws we modify the reference model by feeding back the tracking error signal. Similar approach was used in [7] to improve robustness of the system with respect to disturbances which are bounded by the tracking error. Unfortunately the authors did not further investigate the properties of the control algorithm. We use the error feedback method from different perspectives. Our approach, called modified reference model MRAC or MMRAC in short, is motivated by the fact that the initial large error in the control gains generates large transient excursions both in system's control and output signals. Moreover, the output excursion in its turn generates oscillations in control signal. So the idea is to drive the reference model toward the system proportional to the tracking error, thus preventing the system's attempt to aggressively maneuver toward the reference model. As the tracking error is vanished the modified reference model retains its original form. Therefore, the system asymptotically tracks not only the modified reference model, but also the original one. Moreover, the error feedback term determines the dumping in the control signal, increasing of which makes it possible to increase the learning rate for better transient performance without generating oscillations in the system. Meanwhile, very large error feedback gain may increase the overshoot with respect to original reference model. Therefore, both parameters need to be increased simultaneously for better transient performance, which can be made arbitrary close to 
the ideal performance of the known system. A design guideline is provided for the selection of the feedback gain relative to the adaptation rate. The proposed adaptive control method has uniform performance in the sense that the same tracking is achieved for different reference commands and from different initial conditions without re-tuning.

The rest of the paper is organized as follows. Section II presents results from linear systems theory. Section III present the control design and defines the error signals. We analyze the asymptotic properties of the M-MRAC design in Section IV and the transient properties in Section $\mathrm{V}$, where we also discuss the design specifics. A simulation example is presented in Section VI, and some concluding comments are given in Section VII.

\section{PREliminaries From Linear Systems THEORY}

Consider a linear system $y(s)=G(s) u(s)$, where $G(s)$ is a proper stable transfer function. If $u(t) \in \mathcal{L}_{\infty}$ then the following inequality holds

$$
\|y(t)\|_{\mathcal{L}_{\infty}} \leq\|g(t)\|_{\mathcal{L}_{1}}\|u(t)\|_{\mathcal{L}_{\infty}},
$$

where $g(t)$ is the impulse response of $G(s)$ (see for example [14] (p. 110)). If $u(t) \in \mathcal{L}_{2}$ then we have [14] (p. 108)

$$
\|y(t)\|_{\mathcal{L}_{2}}=\|G(s)\|_{\mathcal{H}_{\infty}}\|u(t)\|_{\mathcal{L}_{2}},
$$

Also, $\|G(s)\|_{\mathcal{H}_{\infty}}$ and $\|g(t)\|_{\mathcal{L}_{1}}$ satisfy the relationship (see Theorem 4.5 in [14])

$$
\|G(s)\|_{\mathcal{H}_{\infty}} \leq\|g(t)\|_{\mathcal{L}_{1}} \leq 2\|G(s)\|_{\mathcal{H}_{\infty}} .
$$

Next, for each $\omega$, we minimize the $\mathcal{H}_{\infty}$ norms of the second order transfer functions

$$
\begin{aligned}
& G_{1}(s)=\frac{1}{s^{2}+2 \zeta \omega s+\omega^{2}} \\
& G_{2}(s)=\frac{s+2 \zeta \omega}{s^{2}+2 \zeta \omega s+\omega^{2}}
\end{aligned}
$$

with respect to $\zeta$. Computation of the $\left\|G_{1}(s)\right\|_{\mathcal{H}_{\infty}}$ results in

$$
\left\|G_{1}\right\|_{\mathcal{H}_{\infty}}=\left\{\begin{array}{ll}
\frac{1}{\omega^{2}}, & \text { if } \quad \zeta>\frac{1}{\sqrt{2}} \\
\frac{1}{2 \omega^{2} \zeta \sqrt{1-\zeta^{2}}}, & \text { if } \quad \zeta \leq \frac{1}{\sqrt{2}}
\end{array},\right.
$$

minimum of which is reached at $\zeta=\frac{1}{\sqrt{2}}$ and is equal to $\left\|G_{1}\right\|_{\mathcal{H}_{\infty}}=\frac{1}{\omega^{2}}$. The $\mathcal{H}_{\infty}$ norm $G_{2}(s)$ is computed to be

$$
\left\|G_{2}\right\|_{\mathcal{H}_{\infty}}= \begin{cases}\frac{2 \zeta}{\omega}, & \zeta>\frac{\sqrt{1+\sqrt{2}}}{2} \\ \frac{1}{\sqrt{2 \omega^{2}\left(\sqrt{1+8 \zeta^{2}}-2 \zeta^{2}-1\right)}}, & \zeta \leq \frac{\sqrt{1+\sqrt{2}}}{2}\end{cases}
$$

minimum of which is reached at $\zeta=\frac{\sqrt{6}}{4}$ and is equal to $\left\|G_{2}\right\|_{\mathcal{H}_{\infty}}=\frac{\sqrt{2}}{\omega}$. We notice that the functions $\left\|G_{1}\right\|_{\mathcal{H}_{\infty}}$ and $\left\|G_{2}\right\|_{\mathcal{H}_{\infty}}$ reach the minimum with respect to $\zeta$ at different points, that is there is no optimal value of $\zeta$ independent of $\omega$ that simultaneously minimizes both $\left\|G_{1}\right\|_{\mathcal{H}_{\infty}}$ and $\left\|G_{2}\right\|_{\mathcal{H}_{\infty}}$. Instead, the whole interval $\frac{\sqrt{6}}{4} \leq \zeta \leq \frac{1}{\sqrt{2}}$ represents suboptimal values for $\zeta$, which results in

$$
\frac{1}{\omega^{2}} \leq\left\|G_{1}\right\|_{\mathcal{H}_{\infty}} \leq \frac{1.0328}{\omega^{2}}, \frac{1.4142}{\omega} \leq\left\|G_{2}\right\|_{\mathcal{H}_{\infty}} \leq \frac{1.4553}{\omega^{2}} .
$$

On the other hand, it is possible to minimize a combination of $\left\|G_{1}\right\|_{\mathcal{H}_{\infty}}$ and $\left\|G_{2}\right\|_{\mathcal{H}_{\infty}}$ that results in $\omega$ independent minimizing $\zeta$. One such combination is $h(\zeta, n)=$ $\left\|G_{1}\right\|_{\mathcal{H}_{\infty}}^{n}+\left\|G_{2}\right\|_{\mathcal{H}_{\infty}}^{2 n}$, where $n$ is a positive integer. Since $h(\zeta)$ is homogeneous in $\omega$, the minimizing value of $\zeta$ is independent of $\omega$. The minimum problem for $n=1$ results in suboptimal values, which are close to the individual optimal values

$$
\zeta_{0}=0.634,\left\|G_{1}(s)\right\|_{\mathcal{H}_{\infty}}=\frac{c_{1}}{\omega^{2}},\left\|G_{2}(s)\right\|_{\mathcal{H}_{\infty}}=\frac{c_{2}}{\omega} .
$$

where $c_{1}=1.0198$ and $c_{2}=1.4162$.

\section{REFERENCE MOdEL MOdIFICATION}

Consider a first order linear system

$$
\dot{x}(t)=a x(t)+b u(t), \quad x(0)=x_{0},
$$

where $x(t) \in \mathbb{R}$ and $u(t) \in \mathbb{R}$ are the output and input of the system, and $a$ and $b$ are unknown constant parameters with known sign of $b$. To simplify notations and without loss of generality we assume that $b>0$. In conventional model reference adaptive control (MRAC) framework, the objective is to design a control signal $u(t)$ such that the output of the system tracks the output $x^{0}(t)$ of the reference model

$$
\dot{x}^{0}(t)=-a_{m} x^{0}(t)+b_{m} r(t), \quad x^{0}(0)=x_{0},
$$

where $a_{m}>0$ and $b_{m}$ are chosen to meet the performance specifications, and $r(t)$ is a smooth and bounded external command. We notice that this reference model results from the application of the ideal control signal

$$
u^{0}(t)=k_{1}^{*} x^{0}(t)+k_{2}^{*} r(t),
$$

where the ideal control gains $k_{1}^{*}$ and $k_{2}^{*}$ satisfy the matching conditions

$$
b k_{1}^{*}=-a-a_{m}, \quad b k_{2}^{*}=b_{m} .
$$

The reference model (9) always can be specified from the performance perspective, but the ideal control signal (10) can not be implemented since the parameters $a$ and $b$ are unknown. Therefore, in MRAC framework the adaptive version of the ideal control signal is implemented, that is

$$
u(t)=\hat{k}_{1}(t) x(t)+\hat{k}_{2}(t) r(t),
$$

where $\hat{k}_{1}(t)$ and $\hat{k}_{2}(t)$ are the estimates of the ideal control gains $k_{1}^{*}$ and $k_{2}^{*}$, and are generated by the adaptive laws

$$
\begin{array}{ll}
\dot{\hat{k}}_{1}(t)=-\gamma x(t) e^{0}(t), & \hat{k}_{1}(0)=k_{10} \\
\dot{\hat{k}}_{2}(t)=-\gamma r(t) e^{0}(t), & \hat{k}_{2}(0)=k_{20},
\end{array}
$$

where $\gamma>0$ is the adaptation rate, and $e^{0}(t)=x(t)-x^{0}(t)$ is the tracking error. Introducing the estimation errors as $\tilde{k}_{1}(t)=$ $\hat{k}_{1}(t)-k_{1}^{*}$ and $\tilde{k}_{2}(t)=\hat{k}_{2}(t)-k_{2}^{*}$, and taking into account the matching conditions (11), we obtain the error dynamics as

$$
\dot{e}^{0}(t)=-a_{m} e^{0}(t)+b\left[\tilde{k}_{1}(t) x(t)+\tilde{k}_{2}(t) r(t)\right] .
$$

It is well known that the this control architecture guarantees asymptotic tracking $x(t) \rightarrow x^{0}(t)$ as $t \rightarrow \infty$. However, the 
transient behavior of $x(t)$ and $u(t)$ cannot be guaranteed. The reason is that high frequency oscillations are generated in the control signal, when the adaptation rate is increased in order to obtain better tracking in transient.

Next we present M-MRAC architecture. We modify the reference model by driving it proportionally to the tracking error toward the system during the transient phase, when the adaptive control signal $u(t)$ is far from the ideal one because of the initial errors in the control gains. Thus, we design the adaptive control based on the modified reference model

$$
\dot{x}_{m}(t)=-a_{m} x_{m}(t)+b_{m} r(t)+\lambda e(t), \quad x_{m}(0)=x_{0},
$$

where $\lambda>0$ is a design parameter and $e(t)=x(t)-x_{m}(t)$. The $e$-dynamics are derived similar to the MRAC architecture and have the form

$$
\dot{e}(t)=-\left(a_{m}+\lambda\right) e(t)+b\left[u(t)-k_{1}^{*} x(t)+k_{2}^{*} r(t)\right] .
$$

The control signal $u(t)$ is still given by the equation (12) as in the MRAC case, but the adaptive laws now are based on the error signal $e(t)$

$$
\begin{array}{ll}
\dot{\hat{k}}_{1}(t)=-\gamma x(t) e(t), & \hat{k}_{1}(0)=k_{10} \\
\dot{\hat{k}}_{2}(t)=-\gamma r(t) e(t), & \hat{k}_{2}(0)=k_{20},
\end{array}
$$

Substituting the control signal $u(t)$ into equation (18) we obtain

$$
\dot{e}(t)=-\left(a_{m}+\lambda\right) e(t)+b\left[\tilde{k}_{1}(t) x(t)+\tilde{k}_{2}(t) r(t)\right],
$$

where the parameter estimation errors $\tilde{k}_{1}(t)$ and $\tilde{k}_{2}(t)$ are defined similar to MRAC case.

In the following analysis we will also need the control error that is defined as $\tilde{u}(t)=u(t)-u^{0}(t)$. From the above constructions it follows that

$$
\tilde{u}(t)=\tilde{k}_{1}(t) x(t)+\tilde{k}_{2}(t) r(t)+k_{1}^{*} e^{0}(t) .
$$

Since the ideal control signal is the best achievable signal, we are interested in minimizing the control error $\tilde{u}(t)$, as well as the tracking error $e^{0}(t)$, both in transient and steady state by selecting proper values for the parameters $\gamma$ and $\lambda$. This is the main objective of the analysis in the following sections.

Remark 3.1: When $\lambda=0$ the proposed control architecture is identical with the conventional MRAC design.

\section{ASYMPTOTIC PROPERTIES OF M-MRAC}

The following theorem summarizes the asymptotic properties of the M-MRAC architecture.

Theorem 4.1: Let the system (8) be controlled by the MMRAC scheme given by (12), (15) and (17). Then

1) all closed-loop signals are bounded

2) $e(t) \rightarrow 0, e^{0}(t) \rightarrow 0$ and $\tilde{u}(t) \rightarrow 0$ as $t \rightarrow \infty$.

Proof: Consider the following candidate Lyapunov function

$$
V(t)=\frac{1}{2} e^{2}(t)+\frac{b}{2 \gamma}\left[\tilde{k}_{1}^{2}(t)+\tilde{k}_{2}^{2}(t)\right] .
$$

Computing its derivative along the trajectories of the systems (18) and (17) we readily obtain

$$
\dot{V}(t)=-\left(a_{m}+\lambda\right) e^{2}(t),
$$

which implies that $e(t), \tilde{k}_{1}(t), \tilde{k}_{2}(t) \in \mathcal{L}_{\infty}$ and $V(t) \in \mathcal{L}_{\infty}$. Since the reference model (15) can be viewed as an exponentially stable system with bounded inputs $r(t)$ and $e(t)$, it follows that $x_{m}(t) \in \mathcal{L}_{\infty}$ as well. Hence $x(t) \in \mathcal{L}_{\infty}$. The boundedness of the tracking error $e^{0}(t)$ follows from the boundedness of $x(t)$ and $x^{0}(t)$. Since the inclusions $\tilde{k}_{1}(t), \tilde{k}_{2}(t) \in \mathcal{L}_{\infty}$ imply that $\hat{k}_{1}(t), \hat{k}_{2}(t) \in \mathcal{L}_{\infty}$, it follows that $u(t) \in \mathcal{L}_{\infty}$. Then $\tilde{u}(t) \in \mathcal{L}_{\infty}$, since $u^{0}(t) \in \mathcal{L}_{\infty}$ by the definition in (10). This completes the first part.

Integrating the equation (21) we obtain

$$
\left(a_{m}+\lambda\right) \int_{0}^{t} e^{2}(\tau) d \tau=V(0)-V(t) .
$$

Since $V(t) \in \mathcal{L}_{\infty}$, it follows that $e(t) \in \mathcal{L}_{2}$. Also, from the error dynamics (18) it follows that $\dot{e}(t) \in \mathcal{L}_{\infty}$, therefore $e(t)$ is uniformly continuous. Application of Barbalat's lemma ( [10], p.19) results in $e(t) \rightarrow 0$ as $t \rightarrow \infty$. Subtracting the equation (14) from the equation (18) we can write

$$
\frac{d}{d t}\left[e(t)-e^{0}(t)\right]=-a_{m}\left[e(t)-e^{0}(t)\right]-\lambda e(t),
$$

Since $e(t) \rightarrow 0$, it follows from the equation (23) that $\left[e(t)-e^{0}(t)\right] \rightarrow 0$, and hence $e^{0}(t) \rightarrow 0$ as $t \rightarrow \infty$. Further, from the dynamic equation (8) it follows that $\dot{x}(t) \in \mathcal{L}_{\infty}$, since $x(t) \in \mathcal{L}_{\infty}$ and $u(t) \in \mathcal{L}_{\infty}$. Therefore, $x(t)$ is uniformly continuous. By assumption $r(t)$ has a bounded derivative, hence is uniformly continuous. From the adaptive laws (17) it follows that $\dot{\tilde{k}}_{1}(t), \quad \dot{\tilde{k}}_{2}(t) \in \mathcal{L}_{\infty}$, therefore $\tilde{k}_{1}(t), \tilde{k}_{2}(t)$ are uniformly continuous as well. Then the right hand side of the error dynamics (18) is uniformly continuous, therefore $\dot{e}(t)$ is uniformly continuous. Since $e(t)$ has a finite limit, it follows from Barabalat's lemma [5] that $\dot{e}(t) \rightarrow 0$ as $t \rightarrow \infty$. Therefore the relationship

$$
\lim _{t \rightarrow \infty}\left[\tilde{k}_{1}(t) x(t)+\tilde{k}_{2}(t) r(t)\right]=0,
$$

holds. Then it follows from the equation (19) that $\tilde{u}(t) \rightarrow 0$ as $t \rightarrow \infty$. The proof is complete.

Theorem 4.1 shows that M-MRAC architecture guarantees not only asymptotic tracking of the modified reference model (15), but also the original reference model of the conventional MRAC. That is the asymptotic performances of both conventional MRAC and proposed M-MRAC designs are equivalent. However, as it is shown in the next section the transient performances are quite different.

\section{Transient Properties of M-MRAC}

In this section we show that the proper selection of the design parameters $\gamma$ and $\lambda$ results in controllable and quantifiable transient performance for both output and input signals of the M-MRAC architecture. 
To this end, we recall that Theorem 4.1 assures that the Lyapunov function $V(t)$ is non-increasing. Therefore the following chain of relationships holds

$$
e^{2}(t) \leq 2 V(t) \leq 2 V(0)=\frac{b}{\gamma}\left[\tilde{k}_{1}^{2}(0)+\tilde{k}_{2}^{2}(0)\right],
$$

which implies that

$$
|e(t)| \leq \frac{\sigma}{\sqrt{\gamma}},
$$

where the constant $\sigma$ is defined as $\sigma=\sqrt{b\left[\tilde{k}_{1}^{2}(0)+\tilde{k}_{2}^{2}(0)\right]}$. Since (26) holds uniformly in $t$ we conclude that

$$
\|e(t)\|_{\mathcal{L}_{\infty}} \leq \frac{\sigma}{\sqrt{\gamma}}
$$

Similarly, from the equation (22) the following is obtained

$$
\|e(t)\|_{\mathcal{L}_{2}} \leq \frac{\sigma}{\sqrt{\left(a_{m}+\lambda\right) \gamma}}
$$

To obtain the bounds on the tracking error $e^{0}(t)$ we write the equation (23) in the following operator form

$$
e^{0}(s)=e(s)+\frac{\lambda}{s+a_{m}} e(s) .
$$

The impulse response of the transfer function $G(s)=\frac{\lambda}{s+a_{m}}$ has an $\mathcal{L}_{1}$ norm of $\frac{\lambda}{a_{m}}$. Therefore, it follows from (27) and (1) that

$$
\left\|e^{0}(t)\right\|_{\mathcal{L}_{\infty}} \leq\|e(t)\|_{\mathcal{L}_{\infty}}+\frac{\lambda}{a_{m}}\|e(t)\|_{\mathcal{L}_{\infty}} \leq \frac{\left(a_{m}+\lambda\right) \sigma}{a_{m} \sqrt{\gamma}} .
$$

On the other hand, since $e(t) \in \mathcal{L}_{2}$, it follows from the equation (23) that $e(t)-e^{0}(t) \in \mathcal{L}_{2}$, hence $e^{0}(t) \in \mathcal{L}_{2}$. Then, applying (2) and (28) we obtain

$$
\left\|e^{0}(t)\right\|_{\mathcal{L}_{2}} \leq \frac{\sigma \sqrt{a_{m}+\lambda}}{a_{m} \sqrt{\gamma}} .
$$

From the derived norm bounds in (27), (28), (30) and (31) it follows that the error signals $e(t)$ and $e^{0}(t)$ can be arbitrarily decreased by increasing the adaptation rate $\gamma$. However, increasing $\lambda$ while improves the $\mathcal{L}_{2}$ performance and leaves intact the $\mathcal{L}_{\infty}$ performance of the error signal $e(t)$, also increases the norm bounds on the error signal $e^{0}(t)$. That is $\lambda$ cannot be selected arbitrarily and should be related to $\gamma$ in such a way that (30) and (31) are not increased when increasing $\lambda$. This can be achieved by setting

$$
a_{m}+\lambda=c \sqrt{\gamma},
$$

where $c>0$ is to be selected form the control error perspectives. In this case, we have the following norm bounds

$$
\left\|e^{0}(t)\right\|_{\mathcal{L}_{\infty}} \leq \frac{c \sigma}{a_{m}}, \quad\left\|e^{0}(t)\right\|_{\mathcal{L}_{2}} \leq \frac{c \sigma}{a_{m} \sqrt{a_{m}+\lambda}},
$$

which imply that the oscillations in the error $e^{0}(t)$ are reduced by increasing $\lambda$ and $\gamma$ simultaneously according to equation (32), whereas the overshoot is specified by the choice of $c$.

Next we analyze the transient performance of the control error signal $\tilde{u}(t)$ defined by the equation (19). We notice
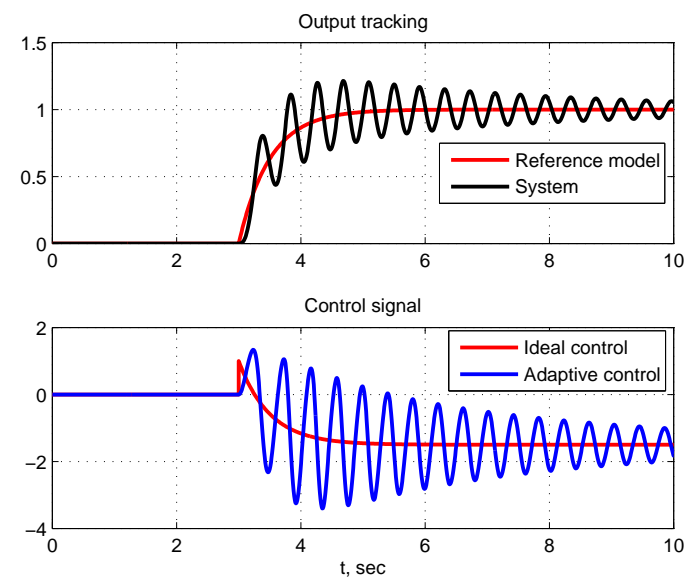

Fig. 1. Response of MRAC to step input with $\gamma=60$.
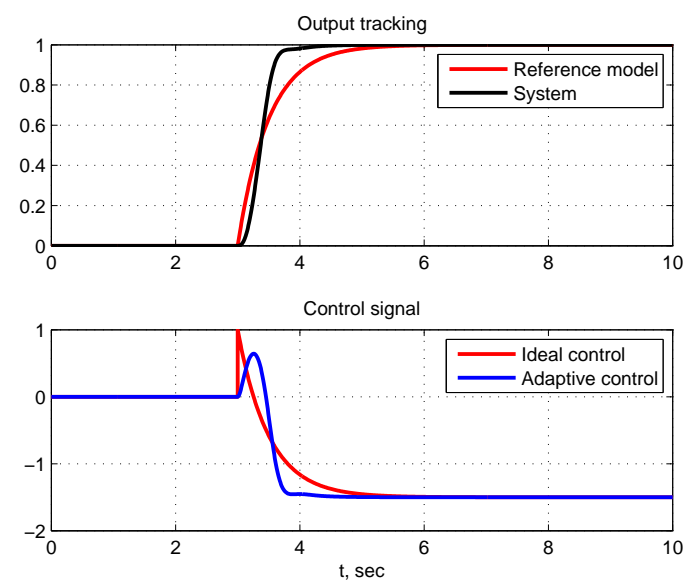

Fig. 2. Response of M-MRAC to step input with $\gamma=60$.

that $\tilde{u}(t)$ does not explicitly depend on design parameters $\lambda$ and $\gamma$. Instead, $\dot{\tilde{u}}(t)$ depend on $\gamma$ through the adaptive laws, and $\ddot{\tilde{u}}(t)$ depend on $\lambda$ through the tracking error dynamics. Differentiating $\tilde{u}(t)$ twice we obtain the following second order differential equation

$$
\begin{gathered}
\ddot{\tilde{u}}(t)+\left(a_{m}+\right) \lambda \dot{\tilde{u}}(t)+\omega^{2}(t) \tilde{u}(t)= \\
\gamma b k_{1}^{*} \rho(t) e^{0}(t)-\gamma \dot{\rho}(t) e(t)+\dot{r}_{1}(t)+\lambda r_{1}(t),
\end{gathered}
$$

where we denote $\rho(t)=x^{2}(t)+r^{2}(t), \omega(t)=\sqrt{\gamma b \rho(t)}$ and $r_{1}(t)=\tilde{k}_{1}(t) \dot{x}(t)+\tilde{k}_{2}(t) \dot{r}(t)+k_{1}^{*} \dot{e}^{0}(t)$. From the results of the previous section it follows that all functions involved in the equation (34) are bounded. In particular, there exist positive constants $\alpha_{1}, \alpha_{2}, \quad \alpha_{3}$ such that $\|\rho(t)\|_{\mathcal{L}_{\infty}} \leq \alpha_{1}$, $\|\dot{\rho}(t)\|_{\mathcal{L}_{\infty}} \leq \alpha_{2}$ and $\left\|r_{1}(t)\right\|_{\mathcal{L}_{\infty}} \leq \alpha_{3}$. Since the terms in equation (34) are also continuous functions in time, it can be considered as a linear equation with time varying coefficients. Although the equation (34) is non-autonomous, it can be still inferred that the adaptation rate $\gamma$ determines the frequency of $\tilde{u}(t)$ and hence the frequency of the control signal $u(t)$, since the ideal control $u^{0}(t)$ is in the low frequency range. 
Therefore, increasing $\gamma$ increases the oscillations in $u(t)$ as it is the case for the conventional MRAC design. On the other hand $a_{m}+\lambda$ determines the damping ratio. Therefore increasing $\lambda$ suppresses the oscillations in $\tilde{u}(t)$ and hence in the control signal $u(t)$. That is, by selecting a proper value for $\lambda$ the desired performance can be achieved. This is the main difference from the MRAC design, which results when $\lambda=0$, since $a_{m}$ is fixed from the performance perspectives.
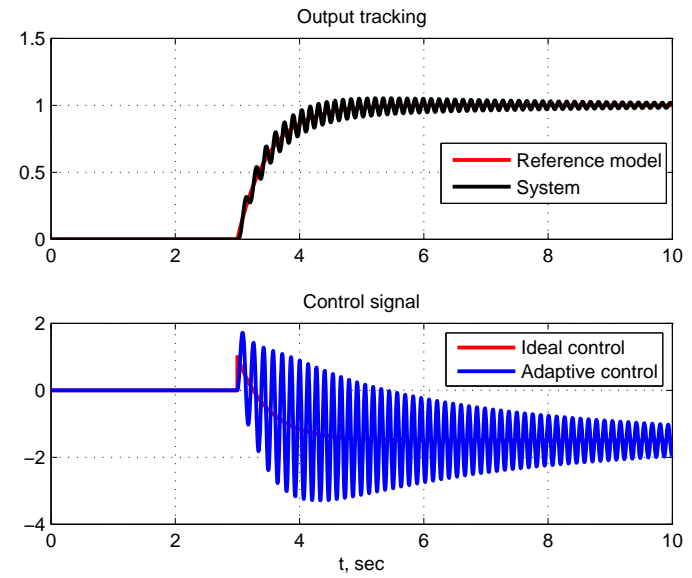

Fig. 3. Response of MRAC to step input with $\gamma=600$.
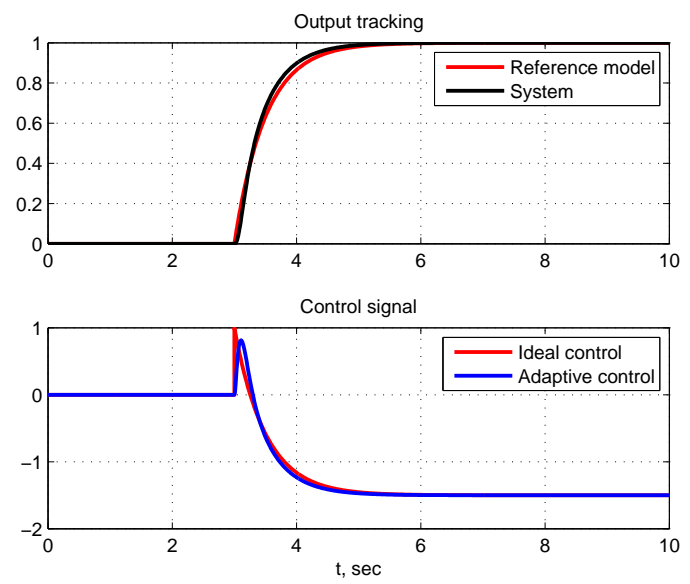

Fig. 4. Response of M-MRAC to step input with $\gamma=600$.

To derive the norm bound for $\tilde{u}(t)$ one needs to compute the state transition matrix of the non-autonomous system (34). However, the analytic computation, even if possible, requires the knowledge of entire function $\omega(t)$. Since our goal is to derive a relationship between $\lambda$ and $\gamma$, that guarantees a suboptimal design, we compute an approximate bound replacing the function $\omega(t)$ with a constant $\omega_{0}$. This is motivated by the fact that the transient behavior of $\tilde{u}(t)$ mainly depends on the initial conditions, and by our interest in the dependence of the bound of $\tilde{u}(t)$ on large values of the parameters $\lambda$ and $\gamma$. That is, when the influence of the parameters $\lambda$ and $\gamma$ is much greater than the influence of other factors. Hence the constant $b$ can be also ignored. Therefore, we set $\omega_{0}=\sqrt{\gamma \rho_{0}}$, where

$$
\rho_{0}=\left\{\begin{array}{l}
\left\|x_{0}\right\|^{2}+\|r(0)\|^{2}, \quad \text { if } r(0) \neq 0 \\
\left\|x_{0}\right\|^{2}+\|r(t)\|_{\mathcal{L}_{\infty}}^{2}, \quad \text { if } \quad r(0)=0, r(t) \neq 0
\end{array}\right.
$$

With constant $\omega_{0}$ the equation (34) can be written in the following operator form

$$
\begin{aligned}
\tilde{u}(s) & =G_{1}(s)\left[\gamma a_{m} \rho(t) e(s)+\gamma k_{1}^{*} \rho(t) e^{0}(t)-\gamma \dot{\rho}(t) e(t)\right. \\
& \left.+\dot{\tilde{u}}(0)-r_{1}(0)\right]+G_{2}(s)\left[r_{1}(t)+\tilde{u}(0)\right]
\end{aligned}
$$

where the transfer functions $G_{1}(s)$ and $G_{2}(s)$ are defined in (4) with $\omega=\omega_{0}$ and $\zeta=\frac{a_{m}+\lambda}{2 \omega_{0}}$. Applying the inequality (1) to each term on the right hand side of the equation (35) we have

$$
\begin{aligned}
\|\tilde{u}(t)\|_{\mathcal{L}_{\infty}} & \leq\left\|g_{1}(t)\right\|_{\mathcal{L}_{1}}\left[\gamma \alpha_{1}\left|k_{1}^{*}\right|\left\|e^{0}(t)\right\|_{\mathcal{L}_{\infty}}+|\dot{\tilde{u}}(0)|\right. \\
& \left.+\gamma \alpha_{2}\|e(t)\|_{\mathcal{L}_{\infty}}+\left|r_{1}(0)\right|\right] \\
& +\left\|g_{2}(t)\right\|_{\mathcal{L}_{1}}\left[\alpha_{3}+|\tilde{u}(0)|\right]
\end{aligned}
$$

Next we select

$$
\lambda=2 \zeta_{0} \sqrt{\gamma \rho_{0}}-a_{m},
$$

where $\zeta_{0}$ is given in (7), and evaluate the right hand side of (37) term by term. Using (27), (33), (3) and (7) we obtain

$$
\begin{gathered}
\left\|g_{1}(t)\right\|_{\mathcal{L}_{1}}\left[|\dot{\tilde{u}}(0)|+\left|r_{1}(0)\right|\right] \leq \frac{2 c_{1}}{\gamma \rho_{0}}\left[|\dot{\tilde{u}}(0)|+\left|r_{1}(0)\right|\right] \\
\gamma \alpha_{1}\left|k_{1}^{*}\right|\left\|g_{1}(t)\right\|_{\mathcal{L}_{1}}\left\|e^{0}(t)\right\|_{\mathcal{L}_{\infty}} \leq \frac{4 c_{1} \alpha_{1}\left|k_{1}^{*}\right| \zeta_{0} \sigma}{a_{m} \sqrt{\rho_{0}}} \\
\gamma \alpha_{2}\left\|g_{1}(t)\right\|_{\mathcal{L}_{1}}\|e(t)\|_{\mathcal{L}_{\infty}} \leq \frac{2 c_{1} \alpha_{2}}{\rho_{0}} \frac{\sigma}{\sqrt{\gamma}} \\
\left\|g_{2}(t)\right\|_{\mathcal{L}_{1}}\left[\alpha_{3}+|\tilde{u}(0)|\right] \leq \frac{2 c_{2}}{\sqrt{\rho_{0} \gamma}}\left[\alpha_{3}+|\tilde{u}(0)|\right]
\end{gathered}
$$

Combining the terms we end up with the following approximate bound for the $\tilde{u}(t)$

$$
\begin{aligned}
\|\tilde{u}(t)\|_{\mathcal{L}_{\infty}} & \leq \frac{4 c_{1} \alpha_{1}\left|k_{1}^{*}\right| \zeta_{0}}{a_{m} \sqrt{\rho_{0}}} \sigma+\frac{2 c_{1} \alpha_{2} \sigma}{\rho_{0} \sqrt{\gamma}} \\
& +\frac{2 c_{2}\left(\alpha_{3}+|\tilde{u}(0)|\right)}{\sqrt{\rho_{0} \gamma}}+\frac{2 c_{1}\left[|\dot{\tilde{u}}(0)|+\left|r_{1}(0)\right|\right]}{\rho_{0} \gamma} .
\end{aligned}
$$

We notice that all the terms on the right hand side of (39) can be arbitrarily decreased by increasing the parameters $\gamma$ except for the first term, which is independent of $\gamma$ and is determined by the initial estimation errors expressed by $\sigma$.

The results can be finalized in the form of the following theorem.

Theorem 5.1: Let the system (8) be controlled by the MMRAC scheme given by (12), (15) and (17). Then for large $\gamma$ and $\lambda$ satisfying the equation (37) the following asymptotic bounds hold

$$
\begin{aligned}
& \|e(t)\|_{\mathcal{L}_{\infty}} \leq o\left(\gamma^{-\frac{1}{2}}\right), \quad\|e(t)\|_{\mathcal{L}_{2}} \leq o\left(\gamma^{-\frac{3}{4}}\right) \\
& \left\|e^{0}(t)\right\|_{\mathcal{L}_{\infty}} \leq o(\sigma), \quad\left\|e^{0}(t)\right\|_{\mathcal{L}_{2}} \leq o\left(\gamma^{-\frac{1}{4}}\right) \\
& \|\tilde{u}(t)\|_{\mathcal{L}_{\infty}} \leq o(\sigma)+o\left(\gamma^{-\frac{1}{2}}\right) .
\end{aligned}
$$




\section{Simulation Results}

For the simulation we consider the system (8) with $a=3$ and $b=2$. The parameters of the reference model are set $a_{m}=2$ and $b_{m}=2$. The external command is chosen to be 1) a step function at time $t=3 \mathrm{sec}$ with the magnitude $r_{0}$, 2) a sinusoid of frequency $2 \mathrm{rad} / \mathrm{sec}$ and of amplitude $r_{0} . \lambda$ is set to $2 \zeta_{0} \sqrt{\gamma\left(x_{0}^{2}+r_{0}^{2}\right)}-a_{m}$ according to equations (35) and (37). The response to the step command of MRAC and M-MRAC with $\gamma=60, r_{0}=1$ and $x_{0}=0$ are displayed in Figures 1 and 2 respectively. The improvement is obvious even with a small adaptation rate. No oscillations are observed in the M-MRAC response contrary to the conventional MRAC response. Next we increase the adaptation rate to $\gamma=600$. The corresponding performances of MRAC and M-MRAC are presented in Figures 3 and 4 respectively. It can be seen that the output tracking performance of MRAC gets better in the expense of high frequency control signal, while the output and input tracking performances of M-MRAC are both perfect. Further increase of $\gamma$ for the M-MRAC design results in the performance indistinguishable from that of the reference model both for input and output signals.
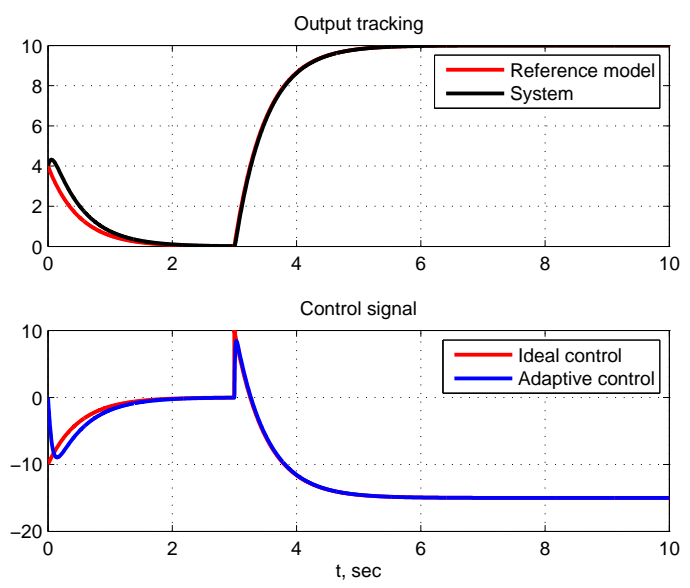

Fig. 5. Response of M-MRAC to step input with $\gamma=6000, r=10$ and $x_{0}=4$.

To show the uniform performance of the M-MRAC design we run simulations with $r_{0}=10$ and $x_{0}=4$, the result of which is displayed on Figure 5. Obviously the quality of the output and input tracking did not change.

The final simulation was performed with the sinusoidal input with $\gamma=6000, r_{0}=1$ and $x_{0}=0$. The systems response along with the M-MRAC control signal is displayed on Figure 5. Ones again perfect tracking is archived in both input and output signals. The noticeable discrepancies disappear with the increase of $\gamma$.

\section{CONCLUSIONS}

We have presented the transient and asymptotic performance analysis of modified reference model MRAC (M-MRAC) design. We showed that the desired level of tracking in both systems input and output signals can be achieved with fast adaptation provided that the design parameters are set according to the derived guideline. Although presented for the conventional MRAC design, the proposed method is suitable for any adaptive design technique since we are only changing the reference model, keeping the controller structure and the adaptive laws intact.
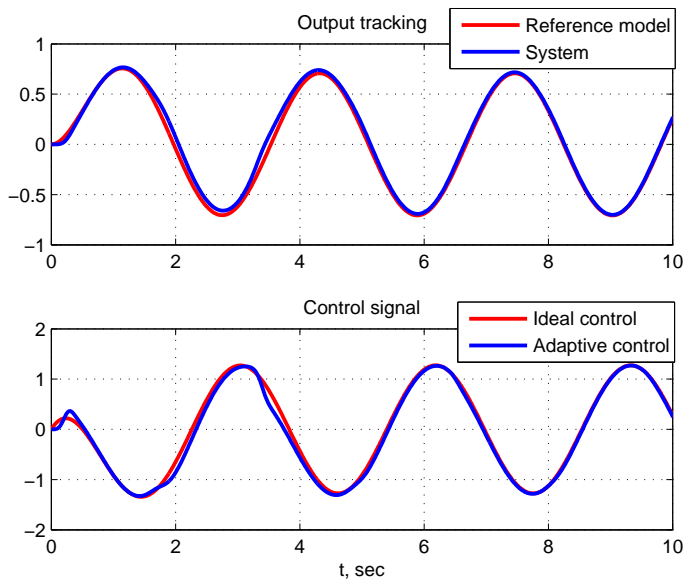

Fig. 6. Response of M-MRAC to sinusoidal input with $\gamma=6000$.

\section{REFERENCES}

[1] A. M. Artega and Y. Tang, "Adaptive Control of Robots with an Improved Transient performance," IEEE Trans. Autom. Contr., vol. 47, no. 7, pp. 1198-1202, 2002.

[2] C. Cao and N. Hovakimyan, "Design and Analysis of a Novel $\mathcal{L}_{1}$ Adaptive Control Architecture with Guaranteed Transient Performance," IEEE Trans. Autom. Contr., vol. 53, no. 2, pp. 586-591, 2008.

[3] A. Datta and M.-T. Ho, "On Modifying Model Reference Adaptive Control Schemes for performance improvement," IEEE Trans. Autom. Contr, vol. 39, no. 9, pp. 1977-1980, 1994.

[4] A. Datta and P. Ioannou, "Performance Analysis and Improvement in Model Reference Adaptive Control," IEEE Trans. Autom. Contr., vol. 39 , no. 12, pp. 2370-2387, 1994.

[5] H. Khalil, Nonlinear Systems, Third Edition. Prentice Hall, New Jersey, 2002.

[6] M. Krstic, I. Kanellakopoulos, and P. Kokotovic, Nonlinear and Adaptive Control Design. John Wiley \& Sons, New York, 1995.

[7] T.-G. Lee and U.-Y. Huh, "An Error Feedback Model Based Adaptive Controller for Nonlinear Systems," In Proc. of IEEE International Symposium on Industrial Electronics, vol. 3, pp. 1095 - 1100, 1997.

[8] S. Morse, "Supervisory Control of Families of Linear Set-Point Controllers - Part 1: Exact Matching," IEEE Trans. Autom. Contr., vol. 41, no. 10, pp. 1413-1431, 1996.

[9] — "Supervisory Control of Families of Linear Set-Point Controllers - Part 1: Robustness," IEEE Trans. Autom. Contr., vol. 42, no. 11, pp. $1500-1515,1997$.

[10] S. S. Sastry and M. Bodson, Adaptive Control: Stability, Convergence and Robustness. Prentice Hall, 1989.

[11] V. Stepanyan, K. Krishnakumar, and N. Nguyen, "Transient Performance and Asymptotic Tracking with Filtering Robust Adaptive Control," In Proc. of the IEEE Aerospace Conference, Big Sky, MT, 2009.

[12] J. Sun, "A Modified Model Reference Adaptive Control Schemes for performance improvement," IEEE Trans. Autom. Contr., vol. 38, no. 7, pp. 1255-1259, 1993.

[13] Z. Zang and R. Bitmead, "Transient bounds for adaptive control systems," In Proceedings of the 30th IEEE Conference on Decision and Control, pp. 2724-2729, December 1990.

[14] K. Zhou, J. Doyle, and K. Glover, Robust and Optimal Control. Prentice Hall, Inc., NJ, 1996. 